\title{
On Finite Generability of Clones of Finite Posets
}

\author{
Ádám Kunos ${ }^{1}\left[\right.$. Miklós Maróti ${ }^{1}$ - László Zádori ${ }^{1}$
}

Received: 11 December 2017 / Accepted: 12 March 2019 / Published online: 4 April 2019

(C) The Author(s) 2019, corrected publication 2019

\begin{abstract}
In the first part of this paper we present a new family of finite bounded posets whose clones of monotone operations are not finitely generated. The proofs of these results are analogues of those in the famous paper of Tardos. Another interesting family of finite posets from the finite generability point of view is the family of locked crowns. To decide whether the clone of a locked crown where the crown is of at least six elements is finitely generated or not one needs to go beyond the scope of Tardos's proof. Although our investigations are not conclusive in this direction, they led to the results in the second part of the paper. We call a monotone operation ascending if it is greater than or equal to some projection. We prove that the clones of bounded posets are generated by certain ascending idempotent monotone operations and the 0 and 1 constant operations. A consequence of this result is that if the clone of ascending idempotent operations of a finite bounded poset is finitely generated, then its clone is finitely generated as well. We provide an example of a half bounded finite poset whose clone of ascending idempotent operations is finitely generated but whose clone is not finitely generated. Another interesting consequence of our result is that if the clone of a finite bounded poset is finitely generated, then it has a three element generating set that consists of an ascending idempotent monotone operation and the 0 and 1 constant operations.
\end{abstract}

Keywords Maximal clones $\cdot$ Finitely generated clones $\cdot$ Bounded posets

The original version of this article was revised due to a retrospective Open Access order.

The authors were supported by the Ministry of Human Capacities 20391-3/2018/FEKUSTRAT, by the National Research, Development and Innovation Office K115518, and by the EU-funded EFOP-3.6.1-16-2016-00008.

Ádám Kunos

akunos@math.u-szeged.hu

Miklós Maróti

mmaroti@math.u-szeged.hu

László Zádori

zadori@math.u-szeged.hu

1 Bolyai Institute, University of Szeged, Aradi vértanúk tere 1, Szeged, 6720, Hungary 


\section{Introduction}

Let $F$ be a set of operations on a set $A$. We call $F$ a clone if it is closed under composition and contains the projections. A subset of a clone is called a subclone if it is closed under composition and contains the projections. On a set $A$ the subclones of the clone of all operations of $A$ form a lattice, the lattice of clones on $A$.

A generating set of a clone $F$ is a subset of $F$ from which every element of $F$ is obtained by the use of composition and projections. A clone is finitely generated if it has a finite generating set. In the present paper we study certain clones related to finite posets. Our main goal is to decide if these clones are finitely generated.

We say that an $n$-ary operation $f$ on $A$ preserves a $k$-ary relation $R$ on $A$, if by applying $f$ componentwise to any $r_{1}, \ldots, r_{n} \in R$ the resulting $k$-tuple also is in $R$. Clearly, for any set of relations $S$ on $A$, the set of operations that preserve all of the relations of $S$ is a clone. The operations that preserve the one element subsets of their base sets are called idempotent.

Let $P$ be a partially ordered set, a poset for short. An operation $f$ on the base set of $P$ is called monotone if $f$ preserves the ordering $\leq$ of $P$. Then we also say that $P$ admits the operation $f$. For a finite poset $P$, let $\mathscr{C}(P)$ and $\mathscr{I}(P)$ denote the clone of monotone operations of $P$ and the clone of idempotent monotone operations of $P$, respectively. We call $\mathscr{C}(P)$ the clone of $P$ and $\mathscr{I}(P)$ the idempotent clone of $P$.

A clone is called maximal if it is a coatom in the lattice of clones. In [1] Rosenberg proved that there are only six types of maximal clones in the lattice of clones on a finite set. Later the clones of five types of them were shown to be finitely generated. The clones of the sixth type are the clones of bounded posets. A poset is bounded if it has a smallest and a largest element. On the finite generability of clones of bounded posets only partial results were obtained so far.

An $n$-ary operation $f, n \geq 3$, is a near unanimity operation if it satisfies the identities

$$
f(x, y, \ldots, y)=f(y, x, \ldots, y)=\cdots=f(y, y, \ldots, x)=y .
$$

Notice that the near unanimity operations are idempotent. It is well known that on a finite set any clone that contains an $n$-ary near unanimity operation is finitely generated. In [2] Demetrovics, Hannák and Rónyai proved that by deleting any convex subset of a finite lattice we obtain a poset whose clone contains a near unanimity operation. A fence is a finite poset of height 1 whose covering graph is a path. The linear sum $P+Q$ of two posets $P$ and $Q$ is the poset whose base set is the union of the base sets of $P$ and $Q$, and whose ordering is defined by $a \leq b$ iff either $a \in P$ and $b \in Q$ or $a \leq b$ in $P$ or $a \leq b$ in $Q$. Let k denote the $k$-element antichain. If $F$ is a fence, then $\mathbf{1}+\mathbf{2}+F+\mathbf{2}+\mathbf{1}$ is a called a locked fence. Fences and locked fences also admit a near unanimity operation. It is easy to see that the class of finite posets whose clones contain near unanimity operations is closed under retract and finite product. A retract of a poset $P$ is a poset $R$ that is isomorphic to the image of a unary monotone operation $f$ on $P$ where $f^{2}=f$.

It is an open question if besides the finite bounded posets that admit a near unanimity operation there are other types of finite bounded posets whose clones are finitely generated. If we drop the boundedness condition in this question, then the answer is positive. A crown is a poset of height 1 whose covering graph is a cycle. In [3] Demetrovics and Rónyai proved that the clone of any crown is finitely generated. It is well known, on the other hand, that the idempotent clone of any crown contains only projections, hence its clone does not contain a near unanimity operation.

In his famous paper [5] Tardos proved that the clone of the eight element poset $T$ in Fig. 1 is not finitely generated. His result was generalized by the third author of the present 
Fig. 1 Posets $T, H$, and $N$
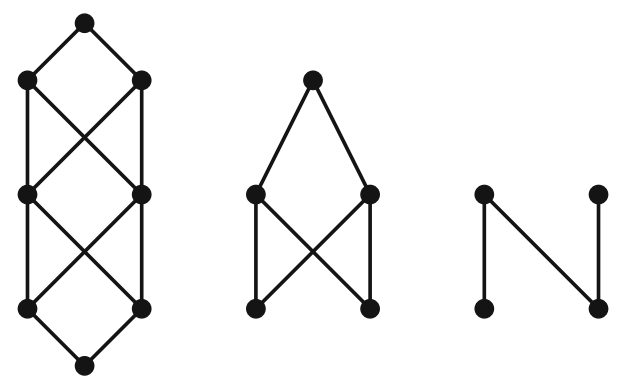

paper in [7]. A finite poset $P$ is series-parallel if the four element poset $N$ in Fig. 1 is not an induced subposet of $P$. In [7] it was proved that for a series-parallel poset $P, \mathscr{C}(P)$ is finitely generated if and only if none of the posets $T, H$ in Fig. 1 and the dual of $H$ are retracts of $P$. A natural question arises: is it true that if the clone of a finite poset is finitely generated, then the clone of any of its retracts is finitely generated. We are not able to answer even the simpler question: is it true that if $T$ or $H$ is a retract of a finite poset $P$, then $\mathscr{C}(P)$ is non-finitely generated.

The aim of this paper is to establish the non-finitely generated (or finitely generated) property for clones of posets in new classes of finite posets. We think that such results eventually may lead to a characterization of finite posets with non-finitely generated clones.

In Section 2 we exhibit an infinite family of finite (bounded) posets which are not seriesparallel and have non-finitely generated clones. Hence we get to new examples of nonfinitely generated maximal clones. Let $A_{n}$ be the poset obtained from the Boolean lattice with $n$ atoms by removing its greatest element, and $B_{n}$ the dual of $A_{n}$. Let $C_{m, n}=A_{m}+$ $\mathbf{2}+B_{n}$ (see Fig. 2). We shall prove that if $m, n \geq 2$, then $\mathscr{C}\left(C_{m, n}\right)$ and $\mathscr{I}\left(C_{m, n}\right)$ are nonfinitely generated. An analogous proof shows that $\mathscr{C}\left(\mathbf{2}+B_{n}\right)$ and $\mathscr{I}\left(\mathbf{2}+B_{n}\right)$ where $n \geq 2$ are not finitely generated. We note that each of the posets $C_{m, n}$ where $m, n \geq 2$ retracts onto $T$, and each of the $2+B_{n}$ where $n \geq 2$ retracts onto $H$.

For any integer $k \geq 2$, let $C_{k}$ denote the $2 k$-element crown. Let $D_{k}$ denote the poset $\mathbf{1}+\mathbf{2}+C_{k}+\mathbf{2}+\mathbf{1}$. These posets were introduced by McKenzie in [4] under the name of locked crowns. To settle the finite generability question for $\mathscr{C}\left(D_{k}\right)$ when $k \geq 3$ seems difficult
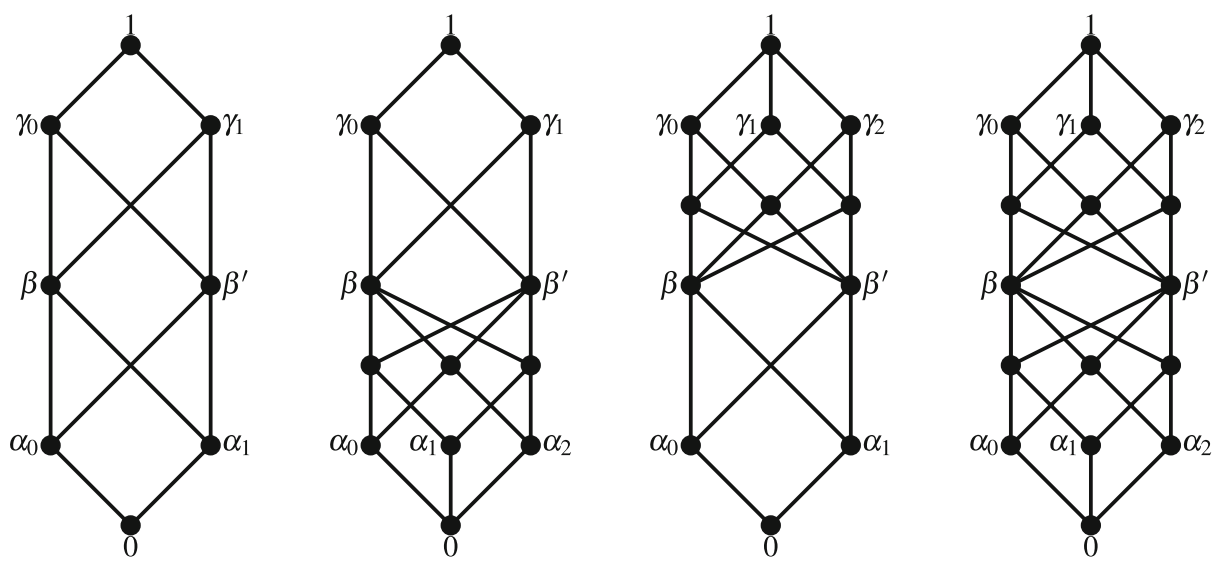

Fig. 2 The posets $C_{2,2}, C_{3,2}, C_{2,3}$, and $C_{3,3}$ 
and needs essentially new ideas beyond the scope of the ones in Tardos's seminal paper [5]. The poset $D_{2}$ is series-parallel and hence, by [7], its clone is non-finitely generated. When $k \geq 3$, then $D_{k}$ is not series-parallel and it is not known whether $\mathscr{C}\left(D_{k}\right)$ is finitely generated or not. Our investigations in this direction led to the results in Section 3.

We call an $n$-ary monotone operation $f$ on a poset ascending if it is greater than or equal to some projection, that is there is an $i$ such that $f\left(x_{1}, \ldots, x_{n}\right) \geq x_{i}$ for all $\left(x_{1}, \ldots, x_{n}\right)$. We prove that the clones of bounded posets are generated by certain ascending idempotent monotone operations and the 0 and 1 constant operations. A consequence of this result is that if the clone of (ascending) idempotent operations of a finite bounded poset is finitely generated, then its clone is finitely generated as well. Another interesting consequence of our result is that if the clone of a finite bounded poset is finitely generated, then it has a three element generating set that consists of an ascending idempotent monotone operation and the 0 and 1 constant operations. Our result does not extend to half bounded finite posets: we prove that the clone of ascending idempotent operations of $H$ is finitely generated but, as we mentioned above, the clone of $H$ is not finitely generated.

Our investigations on the clone of $D_{k}$ led us to seemingly simpler problems. Unfortunately, these problems turned out to be difficult ones, as well. For example, we are not able to decide whether the clone of ascending idempotent operations of $\mathbf{1}+\mathbf{2}+\mathbf{2}+\mathbf{1}$ is finitely generated. Per se, it also remains an open question whether the clone of $D_{k}, k \geq 3$, is finitely generated.

\section{Classes of Finite Posets with Non-Finitely Generated Clones}

In this section we shall prove that the clones and the idempotent clones of the posets $C_{m, n}$, $A_{n}+\mathbf{2}$ and $\mathbf{2}+B_{n}$ where $m, n \geq 2$ are not finitely generated. We require some basic definitions to proceed.

For two posets $O$ and $P$, the partial mappings $f: O \rightarrow P$ are called $P$-colorings of $O$. If $f$ is a $P$-coloring of a poset $O$, then we call the pair $(O, f)$ a $P$-colored poset. The $P$-colored poset $(O, f)$ is called $P$-extendible if there exists a fully defined monotone extension of $f$ to $O$. We say that a poset $O^{\prime}$ is contained in an other poset $O$ if the ordering relation of $O^{\prime}$ is contained in the ordering relation of $O$. A $P$-colored poset $(O, f)$ is called a $P$-obstruction if $(O, f)$ is not extendible, but for all posets $O^{\prime}$ properly contained in $O$, $\left(O^{\prime},\left.f\right|_{O^{\prime}}\right)$ is extendible. An obstruction is trivial if it has two elements or, equivalently, has no non-colored elements. We note that if $O$ is connected, then in the preceding definition it suffices to take those $O^{\prime}$ that are obtained from $O$ by deleting a single covering edge. Clearly, every finite non-extendible colored poset contains an obstruction. Later throughout the text, we frequently use the notation $P \backslash S$ where $P$ is a poset and either $S$ is a subset of elements of $P$ or $S$ only contains a covering edge of $P$. In these cases, $P \backslash S$ denotes the poset remaining from $P$ after removing the elements of $S$ and all edges incident with the elements in $S$, or removing the covering edge of $S$ from $P$, respectively.

First we describe the $B_{n}$-obstructions. By Proposition 1.12 and Theorem 2.2 in [6] each non-trivial $B_{n}$-obstruction consists of a single non-colored element that is covered by the colored elements of the obstruction. By taking into account the definition of obstruction we have the following.

Theorem 1 Every non-trivial $B_{n}$-obstruction consists of a single non-colored element that is covered by the colored elements of the obstruction. The colors of the colored elements 
form an antichain in $B_{n}$ such that their intersection does not exist in $B_{n}$ and the intersection of all but any one of them does exist in $B_{n}$.

Observe that the number of colored elements of a non-trivial $B_{n}$-obstruction is at most $n$, and if the set of colors of a $B_{n}$-obstruction is contained in the set of coatoms of $B_{n}$, then it is equal to it. It also follows that the set of colors of any $B_{n}$-obstruction with $n$-colored elements is equal to the set of coatoms of $B_{n}$. We need the following result, see Theorem 3.3 in [6].

Theorem 2 Let $P$ be a finite poset and $B$ a poset whose obstructions have at most one non-colored element. Let $P^{\prime}=P+B$. Then every non-trivial $P^{\prime}$-obstruction is in one of the following form:

(i) a P-obstruction in which every maximal element is colored,

(ii) a B-obstruction in which every minimal element is colored, or

(iii) it is obtained from a $P$-obstruction $(O, f)$ such that to each non-colored maximal element of $(O, f)$ we glue a B-obstruction with a non-colored minimal element at its minimal element, possibly identifying some colored maximal elements of the same color after the gluing.

We note that part $(i)$ is a special case of part $(i i i)$, when the $P$-obstruction $(O, f)$ has only colored maximal elements. For a more interesting use of part (iii) we provided an example in Fig. 3.

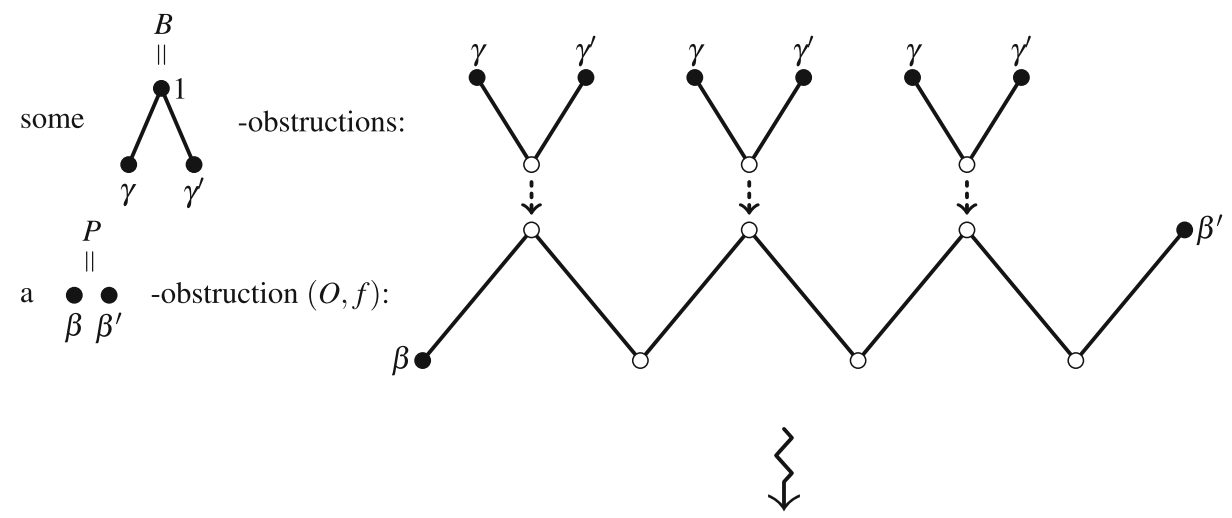

a
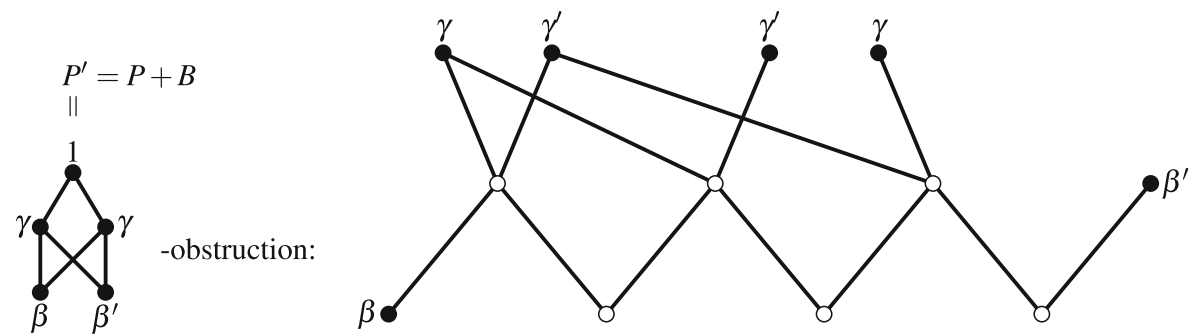

Fig. 3 An example of construction (iii) in Theorem 2 
We remark that the obstructions of the two element antichain $\left\{\beta, \beta^{\prime}\right\}$ are the colored fences whose only colored elements are their two endpoints colored by $\beta$ and $\beta^{\prime}$, respectively.

By this remark, the preceding two theorems and their dual, we obtain a description of the $C_{m, n}$-obstructions. From now on, we refer to the members of the two element antichain in the definition (in the middle) of $C_{m, n}$ as $\beta$ and $\beta^{\prime}$.

Corollary 3 Every non-trivial $C_{m, n}$-obstruction is obtained from a colored fence $(O, f)$ whose endpoints are colored by $\beta$ and $\beta^{\prime}$ such that to each non-colored maximal element of $(O, f)$ we glue a non-trivial $B_{n}$-obstruction and to each non-colored minimal element of $(O, f)$ we glue a non-trivial $A_{m}$-obstruction, possibly identifying some colored maximal elements of the same color and some colored minimal elements of the same color after the gluing.

Now, we are set to prove the main theorem of the section. Our proof is analogous to that of Tardos, hence we advise the reader to consult Tardos's original paper [5] before getting into the proof of our theorem.

Theorem 4 If $m, n \geq 2$, then the clone of $C_{m, n}$ and the idempotent clone $C_{m, n}$ are nonfinitely generated.

Proof First we prove that the clone of $C_{m, n}$ is not finitely generated. For every $k \geq 4$ we shall define a relation $R$ such that all [k/2]-ary monotone operations of $C_{m, n}$ preserve $R$ but there is a monotone operation of $C_{m, n}$ that does not preserve $R$. Then, clearly, for every $k \geq 4, \mathscr{C}\left(C_{m, n}\right)$ is not generated by the [k/2]-ary operations. Thus, $\mathscr{C}\left(C_{m, n}\right)$ is not finitely generated.

The relation $R$ is defined by the help of the poset $Q$ in Fig. 4 . For every $k \geq 4$, poset $Q$ consists of

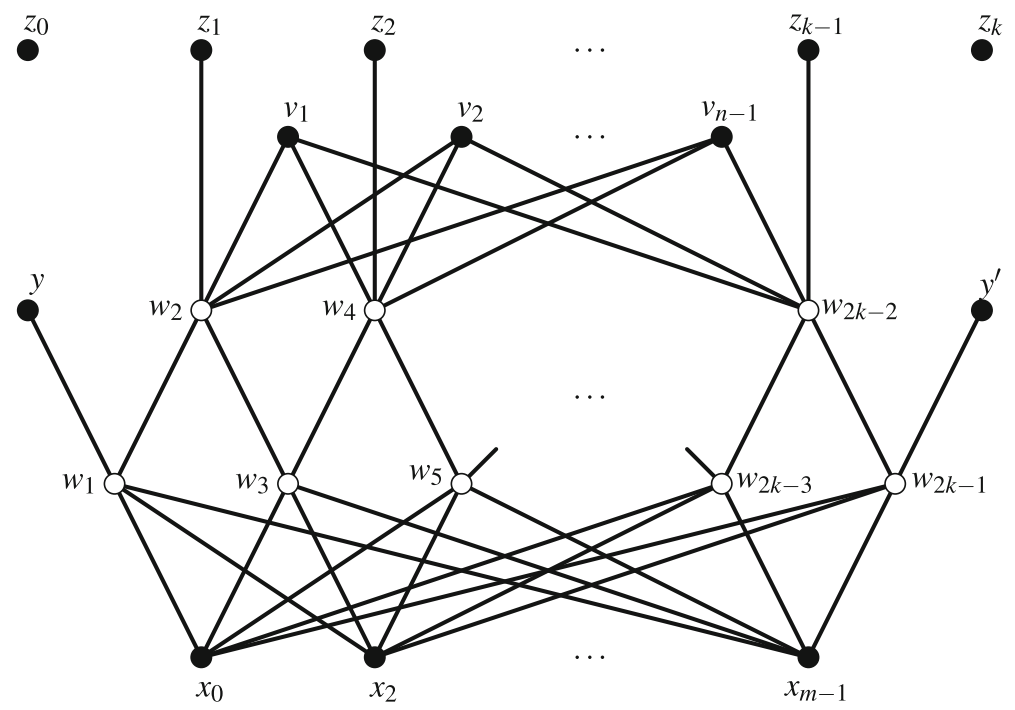

Fig. 4 Poset $Q$ 
(i) the fence $y, w_{1}, w_{2}, \ldots, w_{2 k-1}, y^{\prime}$,

(ii) the minimal elements $x_{0}, x_{2}, \ldots, x_{m-1}$ that are all lower covers of the minimal elements $w_{1}, w_{3}, \ldots, w_{2 k-1}$ of the fence,

(iii) the maximal elements $v_{1}, v_{2}, \ldots, v_{n-1}$ that all cover the maximal elements $w_{2}, w_{4}$, $\ldots, w_{2 k-2}$ of the fence,

(iv) the maximal elements $z_{1}, z_{2}, \ldots, z_{k-1}$ such that $z_{i}$ uniquely covers $w_{2 i}$ for $1 \leq i \leq$ $k-1$, and

(v) two isolated elements $z_{0}$ and $z_{k}$.

Suppose $f$ is a partial map from $Q$ to $C_{m, n}$ whose domain is the set of extremal elements of $Q$. For every $0 \leq j \leq k$ we set $f_{j}\left(z_{i}\right)=f\left(z_{i+j}\right)$ for all $0 \leq i \leq k$ where the indices are meant modulo $k+1$, and $f_{j}(x)=f(x)$ where $x$ is extremal and $x \neq z_{0}, \ldots, z_{k}$.

Now, we define $R_{i}$ to be the ( $\left.m+n+k+2\right)$-ary relation that consists of those partially defined maps $f$ on $Q$ whose domains are the set of extremal elements of $Q,\left(Q \backslash\{e\}, f_{j}\right)$ is extendible for every $0 \leq j \leq k$ and covering edge $e$ of $Q$, and $\left(Q, f_{i}\right)$ is extendible. We note that the $R_{i}$ are preserved by the monotone operations of $C_{m, n}$. Let $R=\cup_{i=0}^{k} R_{i}$. We conceive each element $f \in R$ as an $(m+n+k+2)$-tuple (a column vector) of the form

$$
\left(f\left(x_{0}\right), \ldots, f\left(x_{m-1}\right), f(y), f\left(y^{\prime}\right), f\left(z_{0}\right), \ldots, f\left(z_{k}\right), f\left(v_{1}\right), \ldots, f\left(v_{n-1}\right)\right) .
$$

First, we prove that the $[k / 2]$-ary operations of $C_{m, n}$ preserve $R$. This follows from the fact that for any [k/2] elements in $R$ there is an $i$ such that $R_{i}$ contains all of these elements. To prove this we show that any element $f$ of $R$ is contained by $k-1$ of the $R_{i}$. Suppose that $f$ is in $R$ but not in any of $R_{i_{0}}, R_{i_{1}}$ and $R_{i_{2}}$ where $i_{0}, i_{1}$ and $i_{2}$ are pairwise different indices. This implies that $\left(Q \backslash\left\{z_{0}, z_{k}\right\}, f_{i_{0}}\right.$ ) is an obstruction. Hence - by the use of Corollary 3 , the second remark after Theorem 1 and its dual - up to a symmetry of $C_{m, n}$

$$
\begin{gathered}
f_{i_{0}}\left(x_{0}\right)=\alpha_{0}, \ldots, f_{i_{0}}\left(x_{m-1}\right)=\alpha_{m-1}, f_{i_{0}}(y)=\beta, f_{i_{0}}\left(y^{\prime}\right)=\beta^{\prime}, \\
f_{i_{0}}\left(z_{1}\right)=\cdots=f_{i_{0}}\left(z_{k-1}\right)=\gamma_{0}, f_{i_{0}}\left(v_{1}\right)=\gamma_{1}, \ldots, f_{i}\left(v_{n-1}\right)=\gamma_{n-1}
\end{gathered}
$$

where the $\alpha_{j}$ are the atoms of $A_{m},\left\{\beta, \beta^{\prime}\right\}=\mathbf{2}$ is the two element antichain in the middle of $C_{m, n}$, and the $\gamma_{l}$ are the coatoms of $B_{n}$. We similarly have

$$
f_{i_{1}}\left(z_{1}\right)=\cdots=f_{i_{1}}\left(z_{k-1}\right)=\gamma_{0} \text { and } f_{i_{2}}\left(z_{1}\right)=\cdots=f_{i_{2}}\left(z_{k-1}\right)=\gamma_{0} .
$$

So by the definition of the $f_{i}, f_{i_{0}}\left(z_{0}\right)=f_{i_{0}}\left(z_{k}\right)=\gamma_{0}$ also holds. Hence $f$ is not in any of the $R_{i}$, which contradicts $f \in R$. Thus, $f$ is contained by $k-1$ of the $R_{i}$. Therefore, for any choice of [k/2] elements in $R$ there exists a $j$ such that $R_{j}$ contains them. Hence, any [k/2]-ary monotone operation of $C_{m, n}$ preserves $R$.

Let $g$ be the partial function from $C_{m, n}^{2(k+1)}$ to $C_{m, n}$ defined by the $(k+m+n+2) \times$ $(2 k+3)$-matrix in Fig. 5 such that for each row $g$ assigns the $(2 k+3)$-th component to the $2(k+1)$-tuple determined by the first $2(k+1)$ components of the row. As we mentioned earlier, we conceive each element $f \in R$ as a column vector of the form (1). Notice then that the first $2(k+1)$ columns of the matrix in Fig. 5 are in $R$, and the last column is not in $R$. We shall prove that the colored poset $\left(C_{m, n}^{2(k+1)}, g\right)$ is extendible. Then any extension of $g$ is a monotone $2(k+1)$-ary operation of $C_{m, n}$ that does not preserve $R$, which concludes the proof of the first part of the theorem.

So it remains to prove that $\left(C_{m, n}^{2(k+1)}, g\right)$ is extendible. Suppose that $\left(C_{m, n}^{2(k+1)}, g\right)$ is not extendible. Then it contains an obstruction $\left(O, g^{\prime}\right)$. We invoke Corollary 3 , the first remark after Theorem 1 and its dual. Since $g$ is monotone on its domain, $\left(O, g^{\prime}\right)$ is obtained by adding some suitable colored elements to a colored fence whose endpoints are colored by $\beta$ and $\beta^{\prime}$, respectively. In particular, the endpoints colored by $\beta$ and $\beta^{\prime}$ are maximal in 


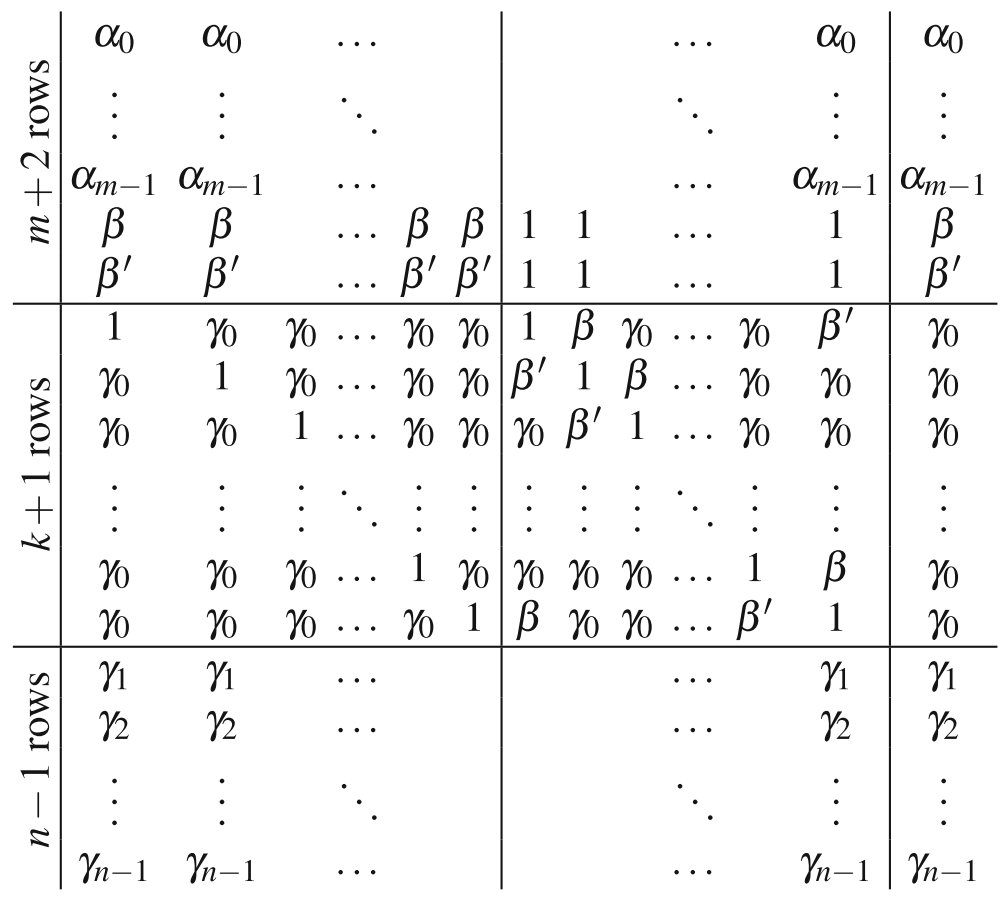

Fig. 5 The matrix defining $g$

$O$, for otherwise one of these elements would be below an element colored by $\gamma_{0}$, which is impossible by the definition of $g$. As the set of colors of $\left(O, g^{\prime}\right)$ is determined by $g$, each minimal non-colored element of the fence has a lower cover colored by $\alpha_{i}$ for all $0 \leq i \leq m-1$ and each maximal non-colored element of the fence has an upper cover colored by a $\gamma_{j}$ for all $0 \leq j \leq n-1$. Observe that all rows with a last component $\gamma_{0}$ from the matrix occur in $\left(O, g^{\prime}\right)$ as $\gamma_{0}$-colored elements. Indeed, if the $l$-th one of them was missing, then the $l$-th projection of $O$ would be an extension of $g^{\prime}$. Let $a_{i}, 1 \leq i \leq t$, be the sequence of $\gamma_{0}$-colored elements in $\left(O, g^{\prime}\right)$ where $a_{i}$ covers the $i$-th maximal non-colored element in the fence of non-colored elements of $\left(O, g^{\prime}\right)$. Let $\left(a_{j}, \gamma_{0}\right)$ the row of the matrix that occurs last in the sequence $\left(a_{i}, \gamma_{0}\right) 1 \leq i \leq t$. Say, $\left(a_{j}, \gamma_{0}\right)$ is the $s$-th row of the matrix. Then the $s-1$-th and the $s+1$-th rows of the matrix occur preceding $\left(a_{j}, \gamma_{0}\right)$ in the sequence $\left(a_{i}, \gamma_{0}\right), 1 \leq i \leq t$. Hence there is a subsequence of consecutive elements of $\left(a_{i}, \gamma_{0}\right), 1 \leq i \leq t$ such that none of the $s-1$-th, $s$-th and $s+1$-th rows occur in it except the first and the last members that coincide with the $s-1$-th and $s+1$-th rows in some order. Here the indices $s-1, s$ and $s+1$ are considered modulo $k+1$. Then, the colored poset whose base poset is $O$ and whose coloring is the restriction of the $(s+k+1)$-th projection to the colored elements of $O$ is a non-extendible colored poset, a contradiction. Thus we have proved that the clone of $C_{m, n}$ is non-finitely generated.

In order to prove that the idempotent clone of $C_{m, n}$ is not finitely generated it suffices to prove that the partial function $g$ given by the matrix in Fig. 5 has a totally defined idempotent monotone extension. First, we extend $g$ by adding the constant $\gamma_{0}$ row to the matrix to obtain a new partial function. The same proof as in the preceding paragraph gives that the partial function defined in this way is extendible. Let $\hat{g}$ be any monotone extension of it onto 
$C_{m, n}^{2(k+1)}$. We claim that the restriction of $\hat{g}$ onto the diagonal tuples must be a projection. The map $\hat{g}$ restricted to the diagonal elements where $g$ is defined and to the constant $\gamma_{0}$-tuple is clearly a projection. The value of $\hat{g}$ on the constant $\beta$-tuple must equal $\beta$ by the definition of $g$ and by the monotonicity of $\hat{g}$. Similarly, on the constant $\beta^{\prime}$-tuple the value of $\hat{g}$ is $\beta^{\prime}$. Then the values of $\hat{g}$ are uniquely determined on the remaining diagonal elements by the monotonicity of $\hat{g}$. Hence, $\hat{g}$ is a projection restricted to the diagonal, so $\hat{g}$ is an idempotent extension of $g$.

Let $Q_{2}$ denote the poset $Q$ from the preceding proof for the parameters $m=n=2$. We note that $Q_{2}$ is the poset used by Tardos in his original proof. One of the anonymous referees pointed out that by using $Q_{2}$ instead of $Q$ for defining $R$ for any $m, n \geq 2$, a similar but a bit simpler proof can be given to prove that the clone of $C_{m, n}$ is non-finitely generated. We have opted for the present proof, since it easily carries over to prove that the idempotent clone of $C_{m, n}$ is non-finitely generated and, in particular, to prove that the idempotent clone of $\mathbf{2}+B_{n}$ is non-finitely generated.

Let $Q^{\prime}$ be the poset obtained from poset $Q$ in the preceding proof by deleting the minimal elements $x_{0}, \ldots, x_{m-1}$. Then $Q^{\prime}$ is used to get a proof of the following theorem. The proof follows mutatis mutandis of the preceding proof, hence we omit it.

Theorem 5 If $n \geq 2$, then the clones $\mathcal{C}\left(2+B_{n}\right)$ and $\mathcal{I}\left(2+B_{n}\right)$ are non-finitely generated.

We note that a similar claim holds for the poset $A_{n}+\mathbf{2}$ if $n \geq 2$. We shall see by Corollary 8 in the next section that if the idempotent clone of a finite bounded poset is finitely generated, then its clone is also finitely generated. By this result, the first part of Theorem 4 implies its second part. We do not know a general result by which the second part of Theorem 5 follows from its first part.

\section{The Clone of Ascending Idempotent Operations}

Recall that a monotone operation of a poset is ascending if it is greater than or equal to some projection. Clearly, the ascending idempotent monotone operations form a subclone in the clone of a poset. In this section we prove a theorem that reduces the finite generability of the clone of a finite bounded poset to the finite generability of the clone of its ascending idempotent operations. We prove that a similar theorem does not hold for half bounded posets. Let $D_{k}$ denote the poset $\mathbf{1}+\mathbf{2}+C_{k}+\mathbf{2}+\mathbf{1}$ where $C_{k}$ is the $2 k$-element crown. We sketch a possible way to prove that the clone of monotone ascending idempotent operations of $D_{k}, k \geq 3$, is non-finitely generated. To decide if $\mathscr{C}\left(D_{k}\right), k \geq 3$, is finitely generated looks further away. An approach like the ones in Tardos's paper and in the proof of Theorem 4 does not seem to work since the shapes of the $D_{k}$-obstructions are too unwieldy due to the fact that the shapes of the $C_{k}$-obstructions are too unwieldy, cf. Theorem 2.

We call the clone of the ascending idempotent operations of a poset the reduced idempotent clone of the poset. The reduced idempotent clone of $P$ is denoted by $\mathscr{I}_{r}(P)$. The following theorem gives indication how ascending idempotent operations play a role in the generability of the clone of a bounded poset.

Theorem 6 The clone of a finite bounded poset is generated by its ascending idempotent operations and the unary constant operations 0 and 1 . 
Fig. 6 The poset $\mathbf{1}+\mathbf{2}+\mathbf{2}+\mathbf{1}$

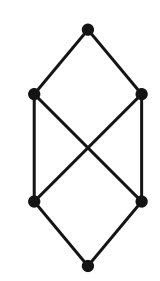

Proof Let $P$ be a finite bounded poset. It suffices to prove that for any monotone $n$-ary $f: P^{n} \rightarrow P$ there exists an ascending idempotent monotone $(n+2)$-ary $f_{I}$ such that $f_{I}\left(0,1, x_{1}, \ldots, x_{n}\right)=f\left(x_{1}, \ldots, x_{n}\right)$. We define $f_{I}$ as follows:

$$
f_{I}\left(y_{1}, y_{2}, x_{1}, x_{2}, \ldots, x_{n}\right):=\left\{\begin{array}{l}
1 \text { if } y_{1} \neq 0 \text { and } y_{2}=1, \\
f\left(x_{1}, \ldots, x_{n}\right) \text { if } y_{1}=0 \text { and } y_{2}=1, \\
y_{1} \text { otherwise. }
\end{array}\right.
$$

Now it is clear that $f_{I}$ is idempotent, monotone, moreover

$$
f_{I}\left(0,1, x_{1}, \ldots, x_{n}\right)=f\left(x_{1}, \ldots, x_{n}\right) \text { and } f_{I}\left(y_{1}, y_{2}, x_{1}, x_{2}, \ldots, x_{n}\right) \geq y_{1} .
$$

The preceding theorem has the following corollaries.

Corollary 7 If the reduced idempotent clone of a finite bounded poset is finitely generated, then its clone is also finitely generated.

Corollary 8 If the idempotent clone of a finite bounded poset is finitely generated, then its clone is also finitely generated.

The first part of Theorem 4 and Corollary 7 immediately yield the following.

Corollary 9 If $m, n \geq 2$, then the clone $\mathscr{I}_{r}\left(C_{m, n}\right)$ is non-finitely generated.

We also note that the first part of Theorem 4 and Corollary 8 implies the second part of Theorem 4.

We do not know if the converse of Corollary 7 is true. The poset $\mathbf{1}+\mathbf{2}+\mathbf{2}+\mathbf{1}$ is a candidate for a counterexample. It is well known that $\mathbf{1}+\mathbf{2}+\mathbf{2}+\mathbf{1}$ admits a 5-ary near unanimity operation, so its clone and idempotent clone are finitely generated. On the other hand, a near unanimity operation on a poset of more than one elements is never ascending. So if the reduced idempotent clone of $\mathbf{1}+\mathbf{2}+\mathbf{2}+\mathbf{1}$ is yet finitely generated, the usual near unanimity argument does not work to prove it. Nevertheless, we are able to prove for a finite bounded poset $P$ that $\mathscr{C}(P)$ is finitely generated if and only if an appropriate subclone of $\mathscr{I}_{r}(P)$ is finitely generated. For a finite bounded poset $P$, let $\mathscr{D}(P)$ denote the clone generated by the ascending idempotent operations defined in the proof of Theorem 6 .

Corollary 10 For a finite bounded poset $P, \mathscr{C}(P)$ is finitely generated if and only if $\mathscr{D}(P)$ is finitely generated.

Proof If $\mathscr{D}(P)$ is finitely generated, then $\mathscr{C}(P)$ is finitely generated by the proof of Theorem 6. For the converse suppose that $\mathscr{C}(P)$ has a finite generating set and is generated 
Fig. 7 Poset $H$ with labeling

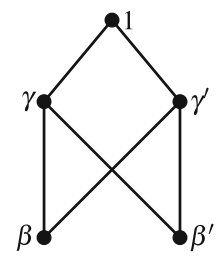

by the operations $f^{1}, \ldots, f^{k}$. Let $f_{I}^{1}, \ldots, f_{I}^{k}$ be the corresponding ascending idempotent operations defined in the proof of Theorem 6.

Now we prove that for any monotone operation $g, g_{I}$ is a composition of $f_{I}^{1}, \ldots, f_{I}^{k}$, hence $\mathscr{D}(P)$ is generated by $f_{I}^{1}, \ldots, f_{I}^{k}$. The operation $g$ is a composition of the operations $f^{1}=f_{I}^{1}(0,1, \ldots), \ldots, f^{k}=f_{I}^{1}(0,1, \ldots)$ where the $\ldots$ within the parentheses stands for a suitable number of variables. By replacing 0 with the variable $y_{1}$ and 1 with the variable $y_{2}$ in this composition, we get to a composition $g^{\prime}$ of $f_{I}^{1}, \ldots, f_{I}^{k}$. By the definition in (2), it is now easy to check that $g^{\prime}=g_{I}$.

Another interesting corollary of Theorem 6 is as follows.

Corollary 11 If the clone of a finite bounded poset is finitely generated, then it is generated by three elements: an ascending idempotent operation and the constant operations 0 and 1.

Proof Let $P$ be a finite bounded poset such that $\mathscr{C}(P)$ is generated by the operations $f^{1}, \ldots, f^{k}$. Then let $f_{I}^{1}, \ldots, f_{I}^{k}$ be the corresponding ascending idempotent operations defined in the proof of Theorem 6 . Then $f_{I}^{1}, \ldots, f_{I}^{k}$ and the 0 and 1 constant operations generate $\mathscr{C}(P)$. Finally, in this generating set we replace $f_{I}^{1}, \ldots, f_{I}^{k}$ by a composition $f$ of them such that $f_{I}^{1}, \ldots, f_{I}^{k}$ are obtained from $f$ by identifying variables. Such an $f$ is defined by replacing two members - say, an $m$-ary $s$ and an $n$-ary $t$ - in the sequence $f_{I}^{1}, \ldots, f_{I}^{k}$ by the operation $s\left(t\left(x_{1}, \ldots, x_{n}\right), \ldots, t\left(x_{(m-1) n+1}, \ldots, x_{m n}\right)\right)$ and by iterating this process until we get to a one element sequence of operations.

It looks as an interesting and non-trivial problem to give some tractable characterization of the finite posets $P$ such that the idempotent operations of $P$ and the constant operations of $P$ together generate the clone of $P$. One of the anonymous referees pointed out that a finite connected poset $P$ with this property must satisfy the fixed point property, that is, every monotone unary operation on $P$ has a fixed point. In this respect, we note that $H$ is a finite connected poset that has the fixed point property, but it is not hard to prove that the idempotent operations and constant operations of $H$ do not generate the clone of $H$. Our next theorem yields the weaker consequence that the ascending idempotent operations and the constant operations of $H$ do not generate the clone of $H$.

We shall prove that the reduced idempotent clone of $H$ is finitely generated. On the other hand, by Theorem 5 the clone of $H$ is not finitely generated. This shows that Corollary 7 does not extend to the class of the half bounded posets. Just to compare, the idempotent clone of $H$ is not finitely generated, also by Theorem 5 .

Theorem 12 The reduced idempotent clone of $H$ is finitely generated. 
This theorem is an immediate consequence of the next two lemmas. We are going to prove that any idempotent operation that is greater than or equal to the first projection is a composition of 4-ary operations of such a type. The whole argument works for the other operations of the reduced idempotent clone analogously.

Let $I r_{1}$ denote the set of the operations in $\mathscr{I}_{r}(H)$ that are greater than or equal to the first projection $\pi_{1}$, and let $I r_{1, n}$ be the $n$-ary part of $I r_{1}$. Next we define some basic operations in $I r_{1}$. Our proof is based on the observation that all members of $I r_{1}$ are built as compositions from these operations.

We say that $f \in I r_{1, n}$ jumps to $q$ at $x \in H^{n}$ if $\pi_{1}(x)<f(x)=q$. We define the smallest operations in $I r_{1, n}$ that jump to a certain value at a certain element. For $a \in H$, let $\underline{a}$ denote the $m$-tuple, each of whose components equals $a$, where $m$ will be clear from the context throughout. Let $z=\left(z_{1}, \ldots, z_{n}\right)$ be an arbitrary element of $H^{n}$.

For any $z$ with $z_{1}<1, z \not \leq \underline{\gamma}$ and $z \not \leq \underline{\gamma^{\prime}}$ we define

$$
g_{1}^{z}(x):=\left\{\begin{array}{l}
1 \text { if } z \leq x, \\
\pi_{1}(x) \text { otherwise. }
\end{array}\right.
$$

For any $z$ with $z_{1}<\gamma$ and $z \not \leq \underline{\gamma^{\prime}}$ we define

$$
g_{\gamma}^{z}(x):=\left\{\begin{array}{l}
\gamma \text { if } z \leq x \text { and } \pi_{1}(x)=z_{1}, \\
1 \text { if } z \leq x \text { and } \pi_{1}(x)=\gamma^{\prime} \\
\pi_{1}(x) \text { otherwise. }
\end{array}\right.
$$

The operation $g_{\gamma^{\prime}}^{z}$ is defined analogously to $g_{\gamma}^{z}$. It is easy to see that $g_{y}^{z} \in I r_{1, n}$ for every possible values of $y$ and $z$. Notice that $g_{y}^{z}$ is the smallest operation in $\operatorname{Ir}_{1, n}$ that jumps to $y$ at $z$.

We define a binary operation denoted by $\vee$ on $H$ that is almost a compatible join semilattice operation:

$$
x \vee y=\left\{\begin{array}{l}
x \text { if }\{x, y\}=\left\{\beta, \beta^{\prime}\right\}, \\
\text { the least upper bound of } x \text { and } y \text { otherwise. }
\end{array}\right.
$$

Obviously, $\vee \in I r_{1,2}$. Moreover, $\vee$ is associative, not commutative, though.

Lemma 13 For any $f \in \operatorname{Ir}_{1, n}$ and $x \in H^{n}$ we have

$$
f(x)=\bigvee\left\{g_{y}^{z}(x): \quad f \text { jumps to } y \text { at } z\right\}
$$

where the order of joinands on the right hand side is chosen arbitrarily.

Proof On one hand for each $x \in H^{n}$ if $y=f(z)>\pi_{1}(z)$, then $g_{y}^{z}(x)$ takes on a value between $\pi_{1}(x)$ and $f(x)$. On the other hand, for each $x$ where $f$ jumps $g_{f(x)}^{x}(x)=f(x)$, so the join on the right hand side of the equality in the claim equals $f(x)$. If $f$ does not jump at $x$, then $g_{y}^{z}(x)=\pi_{1}(x)$ for all of the $g_{y}^{z}$ on the right hand side, and so the join equals $\pi_{1}(x)$.

By Lemma 13, it suffices to exhibit a finite generating set for the operations $g_{y}^{z}$ to finish our proof. The following lemma yields us a generating set of 4 -ary operations. We note that the operations $g_{y}^{z}$ are defined only under some stipulations for the values of the parameters $y$ and $z$, see definition. 
Lemma 14 Let $n \geq 5$. Let $y \in H$ and $z=\left(z_{1}, \ldots, z_{n}\right) \in H^{n}$ such that the $n$-ary operation $g_{y}^{z}$ is defined. Then there exist $i, j$ and $k \neq i, j, 1$ such that for the 4-tuple $z^{\prime}=\left(z_{1}, z_{i}, z_{j}, z_{k}\right)$ and the $(n-1)$-tuple $z^{\prime \prime}=\left(z_{1}, \ldots, z_{k-1}, z_{k+1}, \ldots, z_{n}\right)$, the 4-ary operation $g_{y}^{z^{\prime}}$ and the $(n-1)$-ary operation $g_{y}^{z^{\prime \prime}}$ are defined, and

$$
g_{y}^{z}(x)=g_{y}^{\left(z_{1}, y, y\right)}\left(x_{1}, g_{y}^{z^{\prime}}\left(x^{\prime}\right), g_{y}^{z^{\prime \prime}}\left(x^{\prime \prime}\right)\right)
$$

where $x^{\prime}=\left(x_{1}, x_{i}, x_{j}, x_{k}\right) \in H^{4}$ and $x^{\prime \prime}=\left(x_{1}, \ldots, x_{k-1}, x_{k+1}, \ldots, x_{n}\right) \in H^{n-1}$.

Proof First, we consider the case when $y=1$. Then $z_{1}<1, z \not \leq \gamma$ and $z \not \leq \gamma^{\prime}$. If $z_{i}=1$ for some $i$, then let $j=i$ and choose $k$ to be different from 1 and $i$. If for all $i, z_{i} \neq 1$, then there are two components of $z$ such that one of them equals $\gamma$ and the other does $\gamma^{\prime}$. Then we choose $i, j$ and $k$ such that $z_{i}=\gamma, z_{j}=\gamma^{\prime}$ and $k$ is different from $1, i, j$. In both cases, we take $z^{\prime}$ and $z^{\prime \prime}$ as in the claim. Notice that for the tuples $z^{\prime}$ and $z^{\prime \prime}, g_{1}^{z^{\prime}}$ and $g_{1}^{z^{\prime \prime}}$ are defined. Moreover,

$$
z \leq x \text { iff }\left(z^{\prime} \leq x^{\prime} \text { and } z^{\prime \prime} \leq x^{\prime \prime}\right) .
$$

Thus if $z \leq x$, then $g_{1}^{z^{\prime}}\left(x^{\prime}\right)=1$ and $g_{1}^{z^{\prime \prime}}\left(x^{\prime}\right)=1$, hence

$$
g_{1}^{\left(z_{1}, 1,1\right)}\left(x_{1}, g_{1}^{z^{\prime}}\left(x^{\prime}\right), g_{1}^{z^{\prime \prime}}\left(x^{\prime \prime}\right)\right)=g_{1}^{\left(z_{1}, 1,1\right)}\left(x_{1}, 1,1\right)=1=g_{1}^{z}(x) .
$$

For the case when $z \not \leq x$, we may assume that $x_{1}<1$, since otherwise both sides of the equality in the claim equal 1 . Now if, for example, $z^{\prime}\left\lfloor x^{\prime}\right.$, then $g_{1}^{z^{\prime}}\left(x^{\prime}\right)=x_{1}<1$. This yields

$$
g_{1}^{\left(z_{1}, 1,1\right)}\left(x_{1}, g_{1}^{z^{\prime}}\left(x^{\prime}\right), g_{1}^{z^{\prime \prime}}\left(x^{\prime \prime}\right)\right)=g_{1}^{\left(z_{1}, 1,1\right)}\left(x_{1}, x_{1}, g_{1}^{z^{\prime \prime}}\left(x^{\prime \prime}\right)\right)=x_{1}=g_{1}^{z}(x),
$$

which concludes our proof for the case $y=1$.

For the remaining part of the proof, we assume without loss of generality that $y=\gamma$. Then $z_{1}<\gamma$ and $z \not \leq \gamma^{\prime}$. We may assume that $z_{1}=\beta$. Now, there exists an $i$ such that $z_{i}=1$ or $z_{i}=\gamma$. We put $j=i$ and choose $k$ different from 1 and $i$. We take $z^{\prime}$ and $z^{\prime \prime}$ as in the claim. Then $g_{\gamma}^{z^{\prime}}$ and $g_{\gamma}^{z^{\prime \prime}}$ are defined, and

$$
z \leq x \text { iff }\left(z^{\prime} \leq x^{\prime} \text { and } z^{\prime \prime} \leq x^{\prime \prime}\right)
$$

We split the rest of the proof in three cases.

In the first case we assume that $z \leq x$ and $x_{1}=\beta$. Then we have that $g_{\gamma}^{z^{\prime}}\left(x^{\prime}\right)=\gamma$ and $g_{\gamma}^{z^{\prime \prime}}\left(x^{\prime \prime}\right)=\gamma$, hence

$$
g_{\gamma}^{(\beta, \gamma, \gamma)}\left(x_{1}, g_{\gamma}^{z^{\prime}}\left(x^{\prime}\right), g_{\gamma}^{z^{\prime \prime}}\left(x^{\prime \prime}\right)\right)=g_{\gamma}^{(\beta, \gamma, \gamma)}(\beta, \gamma, \gamma)=\gamma=g_{\gamma}^{z}(x) .
$$

In the second case we assume that $z \leq x$ and $x_{1}=\gamma^{\prime}$. Now we have that $g_{\gamma}^{z^{\prime}}\left(x^{\prime}\right)=1$ and $g_{\gamma}^{z^{\prime \prime}}\left(x^{\prime \prime}\right)=1$, and hence

$$
g_{\gamma}^{(\beta, \gamma, \gamma)}\left(x_{1}, g_{\gamma}^{z^{\prime}}\left(x^{\prime}\right), g_{\gamma}^{z^{\prime \prime}}\left(x^{\prime \prime}\right)\right)=g_{\gamma}^{(\beta, \gamma, \gamma)}\left(\gamma^{\prime}, 1,1\right)=1=g_{\gamma}^{z}(x) .
$$

For the third case we assume that none of the conditions

$$
\left(z \leq x \text { and } x_{1}=\beta\right) \text { and }\left(z \leq x \text { and } x_{1}=\gamma^{\prime}\right)
$$

hold. This implies that if $z \leq x$, then $x_{1}=\gamma$ or $x_{1}=1$, and it is clear in both cases that both sides of the equality in the claim equal $x_{1}$. Hence we have to consider only $z \not \leq x$. Then, for example, $z^{\prime \prime} \not \leq x^{\prime \prime}$ and $g_{\gamma}^{z^{\prime \prime}}\left(x^{\prime \prime}\right)=x_{1}$. This yields

$$
g_{\gamma}^{(\beta, \gamma, \gamma)}\left(x_{1}, g_{\gamma}^{z^{\prime}}\left(x^{\prime}\right), g_{\gamma}^{z^{\prime \prime}}\left(x^{\prime \prime}\right)\right)=g_{\gamma}^{(\beta, \gamma, \gamma)}\left(x_{1}, g_{\gamma}^{z^{\prime}}\left(x^{\prime}\right), x_{1}\right)=x_{1}=g_{\gamma}^{z}(x),
$$

which concludes the proof. 
Finally, we delineate some ideas on the question if $\mathscr{I}_{r}\left(D_{k}\right)$ is finitely generated. We proceed with a straightforward lemma on general clones. A homomorphism from a clone $C$ to a clone $D$ is a map that preserves the projections and commutes with composition of operations. A clone $D$ is a homomorphic image of a clone $C$ if there is an onto homomorphism from $C$ to $D$.

Lemma 15 If a clone is finitely generated, then its homomorphic images are also finitely generated.

Let $P$ be a finite poset. A subset $U$ of $P$ is called an up-set of $P$, if for any $a \in U$, $b \in P$ and $a \leq b$ we have $b \in U$. We note that every $n$-ary monotone ascending idempotent operation of an up-set $U$ of $P$ extends to an $n$-ary monotone ascending idempotent operation on $P$. Indeed, by taking an appropriate projection on $P^{n} \backslash U^{n}$ yields an extension. Moreover, any up-set $U$ of $P$ is preserved by all monotone ascending operations of $P$, hence $\mathscr{I}_{r}(U)$ is a homomorphic image of $\mathscr{I}_{r}(P)$ via the restriction homomorphism. So by Lemma 15 we get the following.

Corollary 16 If the reduced idempotent clone of a finite poset $P$ is finitely generated, then the reduced idempotent clone of any up-set of $P$ is finitely generated.

We mentioned above that we are not able to decide whether $\mathscr{I}_{r}(\mathbf{1}+\mathbf{2}+\mathbf{2}+\mathbf{1})$ is finitely generated. By the preceding corollary - as $\mathbf{1}+\mathbf{2}+\mathbf{2}+\mathbf{1}$ is an up-set in $D_{k}$ - a negative answer would yield that $\mathscr{I}_{r}\left(D_{k}\right)$ is non-finitely generated. We note that $D_{2}$ is series-parallel and $T$ is a retract of it, and hence $\mathscr{C}\left(D_{2}\right)$ is non-finitely generated. So by Corollary $7, \mathscr{I}_{r}\left(D_{2}\right)$ is non-finitely generated. Nevertheless, it remains open whether $\mathscr{I}_{r}\left(D_{k}\right)$ and $\mathscr{C}\left(D_{k}\right)$ are finitely generated if $k \geq 3$.

Acknowledgements Open access funding provided by University of Szeged. We are thankful to the anonymous referees, especially to one of them whose suggestions made the proof of Theorem 4 simpler and led to an improved version of the definition of the operations $g_{y}^{z}$ in the proof of Theorem 12.

Open Access This article is distributed under the terms of the Creative Commons Attribution 4.0 International License (http://creativecommons.org/licenses/by/4.0/), which permits unrestricted use, distribution, and reproduction in any medium, provided you give appropriate credit to the original author(s) and the source, provide a link to the Creative Commons license, and indicate if changes were made.

\section{References}

1. Rosenberg, I.G.: Über die funktionale Vollständingkeit in dem mehrwertigen Logiken. Rozpravy Ceskoslovenske Akad. Ved., Ser. Math. Nat. Sci. 80, 3-93 (1970)

2. Demetrovics, J., Hannák, L., Rónyai, L.: Near unanimity functions of partial orderings. In: Proc. 14th International Symp. on Multiple Valued Logic, Winnipeg, pp. 52-56 (1984)

3. Demetrovics, J., Rónyai, L.: Algebraic properties of crowns and fences. Order 6/1, 91-99 (1989)

4. McKenzie, R.: Monotone clones, residual smallness and congruence distributivity. Bull. Austral. Math. Soc. 41, 283-300 (1990)

5. Tardos, G.: A maximal clone of monotone operations which is not finitely generated. Order 3/3, 211-218 (1986)

6. Zádori, L.: Posets, near unanimity functions and zigzags. Bull. Aust. Math. Soc. 47/1, 79-93 (1993)

7. Zádori, L.: Series-parallel posets with non-finitely generated clones. Order 10/4, 305-316 (1993)

Publisher's Note Springer Nature remains neutral with regard to jurisdictional claims in published maps and institutional affiliations. 